\title{
Historical Summary and Recommendations
} on Melanoma in the LLNL Workforce

Dan H. Moore, II

Fred Hatch

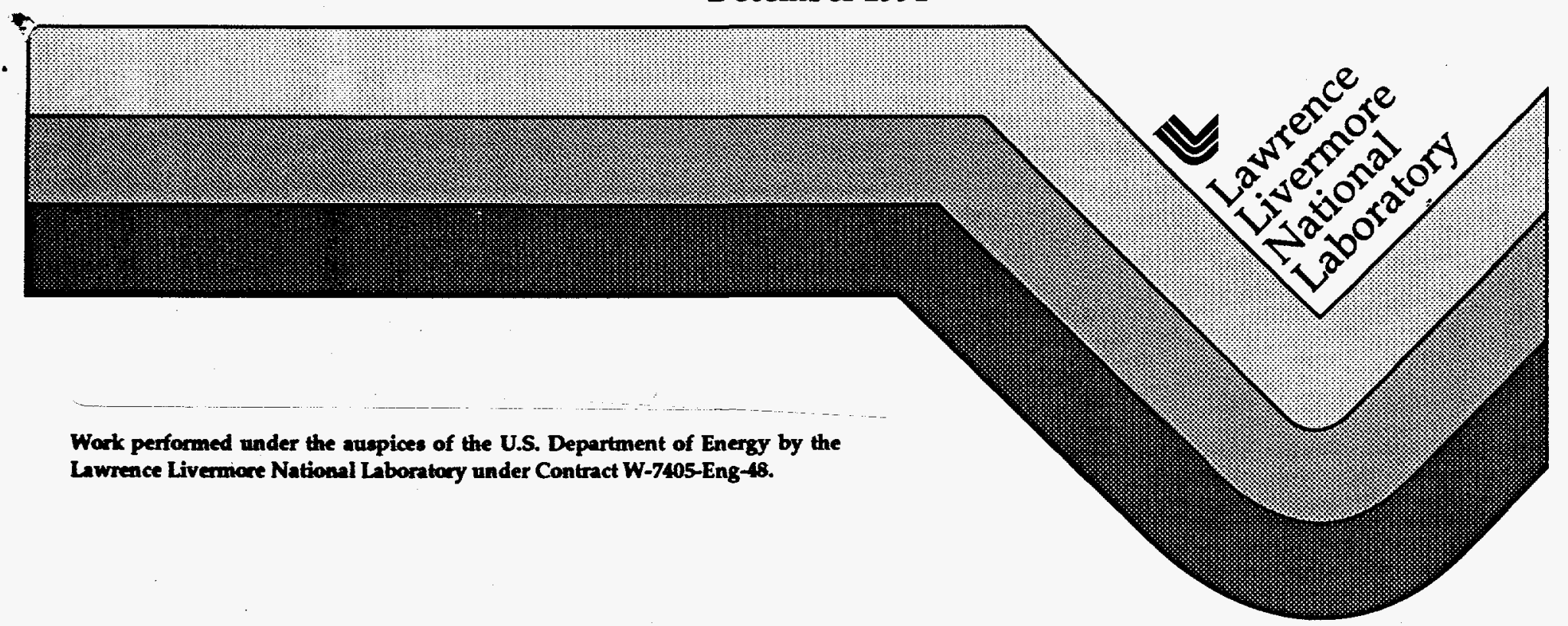




\section{DISCLAIMER}

This report was prepared as an account of work sponsored by an agency of the United States Government. Neither the United States Government nor any agency thereof, nor any of their employees, make any warranty, express or implied, or assumes any legal liability or responsibility for the accuracy, completeness, or usefulness of any information, apparatus, product, or process disclosed, or represents that its use would not infringe privately owned rights. Reference herein to any specific commercial product, process, or service by trade name, trademark, manufacturer, or otherwise does not necessarily constitute or imply its endorsement, recommendation, or favoring by the United States Government or any agency thereof. The views and opinions of authors expressed herein do not necessarily state or reflect those of the United States Government or any agency thereof. 


\section{DISCLAIMER}

Portions of this document may be illegible in electronic image products. Images are produced from the best available original document. 
Historical Summary and Recommendations on Melanoma in the LLNL Workforce

Dan H. Moore II

Fred Hatch

DISTRIBUTION OF THIS DOCUMENT IS UNLIMITED

Page 1

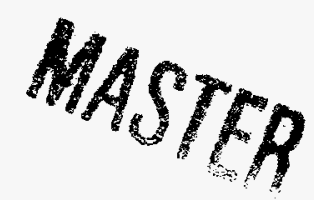




\section{Disclaimer:}

This document was written by the two most recent chairmen of the Melanoma Investigation Task Group at the time of their departure from the Laboratory. It is intended to provide corporate memory, background and advice for LLNL management, oversight committees, and consultants or reviewers who may be called upon to assist the Laboratory. The contents reflect the opinions of the authors and are not statements of Task Group consensus. The recommendations have not yet been accepted, modified, or rejected by Lab Management.

\section{Background and Recognition of Increased Melanoma at LLNL}

Melanoma of the skin comprises about 3.5 percent of the incidence (38000 new cases in 1991) and 1.7 percent of the mortality (8500 deaths in 1991) of all cancer in the United States. However, for several decades it has shown the fastest rate of increase of any cancer site. It is somewhat more common in males than in females in the United States, but elsewhere it is more common in females.

The Laboratory was founded in 1952 and there were no known cases of cutaneous melanoma until one case in 1960, followed by single cases in 1963 and 1964. Starting in 1968 there was one case per year until 1972 when a cluster of four cases occurred. Although there were no cases in 1973, four cases each year were diagnosed in 1974 and 1975, and two cases in 1976. The total was now 21; however, only 15 of these were known to the medical department. Early in 1977 Dr. Max Biggs, the Lab Medical Director, requested assistance from Dr. Donald Austin and the Resource for Cancer Epidemiology (RCE) of the California Department of Health Services, which maintains the Tumor Registry for the San Francisco Bay Area, to investigate the apparent excess of cases among employees. This study was funded by the Laboratory. Drs. Austin and Reynolds, also of the RCE, compared the number of melanoma cases observed among LLNL employees during 1972-1977 with the number expected based upon local (two adjacent counties where most employees lived) age-, race-, sex-, and census tract-specific rates. The results were released in April 1980 as Report No. 1 and later published in The Lancet (Oct. 3, 1981, pp. 712-716). The 19 cases of melanoma diagnosed during the study period among Laboratory employees was determined to be three- to four-fold that expected. The media 
publicity concerning the suspected increase may have contributed to the finding of eleven new cases in 1980. Retrospective analysis of 6-year cumulative data showed that the LLNL incidence rate began to exceed that of the adjacent counties about 1972 .

\section{History of Melanoma Studies at LLNL}

Upon receipt of the Austin-Reynolds report the Laboratory promptly formed a Melanoma Task Group, chaired by Dr. Lowry Dobson, to investigate the problem and monitor the research studies to be performed. In April 1980 the Secretary of Energy formed an ad hoc advisory board to review the data and identify potential causal factors. The Advisory Board, chaired by Dr. Arthur Upton, a former Director of the National Cancer Institute, concluded that the melanoma incidence rate among employees did, in fact, exceed the rate in the local community, but could not implicate any specific cause. The Board noted that "the possibility cannot be excluded that the excess may ultimately prove to reflect the influence of socioeconomic factors and lifestyle, rather than exposure to a cancer-causing agent in the workplace". As a result Dr. Austin's group was further contracted, with Dept. of Energy funding, to conduct a case-control study of LLNL employees and to determine the incidence rates of cancers other than melanoma at LLNL.

Austin's Report No. 2 in October 1982 titled "A Study of Cancer Incidence in Lawrence Livermore National Laboratory Employees" found that cancer in general (all sites except melanoma) during 1969-1980 was not elevated above rates in the Bay Area population. A further report in November 1982 from Austin and Reynolds titled "Familial Risk Factors for Malignant Melanoma of the Skin" identified two factors that might serve as clinical markers of high risk individuals: parental history of skin cancer (non-melanoma), and presence of numerous large moles ( $5 \mathrm{~mm}$ or more in diameter). These findings have since been amply confirmed in the epidemiological literature.

Laboratory melanoma incidence rates were also studied by Dr. Robert Hiatt and Bruce Fireman of the Kaiser Foundation. Their February 1984 report "Malignant Melanoma in the Kaiser Foundation Health Plan of Northern California: A Comparison of Incidence and Measures of Health Care Utilization Between the Lawrence Livermore National Laboratory and Surrounding Health Plan Groups" confirmed the approximately three-fold higher incidence of 
melanoma in LLNL employees than in non-Laboratory Kaiser Plan members residing in the area. A similarly elevated incidence rate was observed in spouses of LLNL employees; however, this was based on only six cases and was not statistically significant. LLNL employee Kaiser Plan members were found to have their skin biopsied for pigmented lesions significantly more often than non-LLNL members. This finding was tentatively ascribed to the awareness of the increased incidence at LLNL by employee members and by Kaiser Plan medical staff.

Austin's case-control Report No. 3 in July 1984 studied 31 cases and 110 matched controls who were interviewed in 1981. Detailed comparisons were made of 180 factors, and multiple linear logistic regression analysis was performed on those factors thought to be relevant to causation. The LLNL cases were reported to have the typical personal and medical characteristics recognized in melanoma epidemiology. In addition, five occupational factors were asserted to explain quantitatively a 3-4-fold increase in incidence. The suggested factors are as follows:

1. Exposure to radioactive materials

2. One or more visits to Site 300

3. Exposure to volatile photographic chemicals

4. Visit to Pacific Test Site during a nuclear test

5. Duties as a chemist.

Dr. Carl Shy of the University of North Carolina School of Public Health assembled a panel for external review of the Austin case-control study from the points of view of epidemiological validity and occupational credibility. This was submitted to LLNL and presented to employees in January 1986. The conclusions of the review follow (in part).

1. The study design and statistical methods were appropriate and properly applied;

2. Because of the small number of cases (31) it is very difficult to identify the independent effects of occupational and non- occupational risk factors;

3. The report of Austin and Reynolds overstated their conclusions about the five occupational factors primarily because experimental evidence linking melanoma causation with these occupational factors is very weak or nonexistent;

4. "A causal relationship between occupational exposures at LLNL and the risk of developing malignant melanoma has not been established"; 
5. Some or all of the excess cases may be explained by intensive surveillance of the employees' moles and a high rate of biopsy of these moles caused by enhanced concern by Laboratory employees and their physicians about the reported melanoma excess.

In February of 1986 an external Working Group, again chaired by Dr. Upton, reviewed the completed studies and plans at LLNL. This group was not entirely convinced that the elevated incidence at LLNL is real, although they could offer only limited help in designing ways to evaluate this. They favored our proposed pathology review studies, and some members wished to have extensive clinical history data gathered on each case. A preliminary proposal from LLNL to carry out an in-house occupational medical-industrial hygiene study in case-control format was not well received. The Working Group made recommendations for sharper focus in questionnaire design and strongly suggested doing only cases. The LLNL Task Group proceeded eventually with this study in a case-control format, despite lack of blessing by the Working Group.

Dr. Lawrence Kupper and associates at the University of North Carolina Dept. of Biostatistics was asked to review the Austin case-control report rigorously for accuracy of the database and appropriateness and accuracy of the statistical calculations; they reported their results to the Laboratory in March 1987 and submitted a complete report in July 1987. After validation in situ of the interview questionnaire database and replication of the Austin multiple logistic regression models, Kupper et al. carried out extensive further studies of their own design, primarily directed at examining the robustness of the imputed occupational factors in the presence of many sets of relevant non-occupational factors derived both from the Austin study and from the epidemiological literature. Three occupational factors remained robust and significant:

1. Working around radioactive materials,

2. Presence at Site 300,

3. Working around volatile photographic chemicals.

Despite the perceived importance of factor no. 1, an analysis of radiation exposure data in far more depth than was possible at the time of Austin's study failed to show any relationship of documented exposure levels with the risk factor "working around radioactive materials. "The melanoma risk enhancement from factors 1 and 2 was stronger in the earlier than in the later part of the study period, with a shift possibly around 1974 . The interpretation favored by Kupper 
et al. was that the three significant occupational factors may be "surrogates" for an unknown hypothetical factor encountered by employees who answered affirmative to some or all of the three surrogate factors. The association of the surrogates with the hypothetical factor may have been true only in the early years; or exposure to the hypothetical factor may have become less prevalent with the passage of time.

Sources of bias in the Austin study were examined and found to be unlikely to have had major effect. Recall bias between cases and controls, such that cases more frequently recall possible exposures, is impossible to eliminate. Kupper et al. present two caveats about the Austin study. First, the large number of statistical tests performed make the probability quite high of falsely declaring a factor or factors to be significant. Such a large number of significance tests is not unusual or inappropriate, so long as the study is viewed as exploratory (hypothesis-generating) and not definitive. Partly on this basis, they suggest that the study was "over interpreted" by Austin and Reynolds. They disagree with the implication of the causal nature of the occupational factors originally identified, but agree that these factors are the best candidates for further investigation. They believe there is a good possibility that ionizing radiation exposure is not a risk factor for melanoma at LLNL, but that this deserves further investigation. We find it necessary to comment that the Kupper report does not examine the biological plausibility of the risk factors, such as the conspicuous lack of linkage of melanoma epidemiology to ionizing radiation. It also does not consider the extensive negative epidemiologic studies on the workforce of Los Alamos National Laboratory, which has great similarity of workplace conditions to LLNL. Biological plausibility and comparative epidemiology were considered more fully in the report by Shy et al. We believe it is important to merge the latter considerations with the more purely statistical analysis when comprehensively analyzing the problem of melanoma in the LLNL workforce. The combined reviews find little fault with the performance of the Austin and Reynolds studies; but they seriously undermine the conclusions on grounds of statistics and credibility.

In early $1991 \mathrm{Dr}$. Robert Hiatt of the Kaiser Foundation reported on a blind review of histopathologic slides by three eminent dermatopathologists. There were 20 LLNL cases diagnosed between 1970 and 1984 and 36 matched control (non-LLNL) cases. The study hypothesis was that intensified surveillance of LLNL employees had resulted in an elevated incidence rate by picking up 
thinner, earlier lesions than were observed in non-LLNL Plan members. The findings support surveillance bias prior to about 1976, which was before widespread recognition and publicity about increased incidence at LLNL. The report stated that data since 1976 showed no difference in lesion thickness and, therefore, would not explain the high incidence in the LLNL population since then. Drs. Moore and Schneider at LLNL disagree with some of the methodological details of this study and have carried out an independent study of lesion thickness, based on a comparison of all LLNL and all Kaiser Walnut Creek melanomas, that does support surveillance bias as possibly contributing to the elevated rate at LLNL during both pre-1976 and post-1976 time periods.

In early 1992 two coordinated studies were reported dealing with lesion thickness and with the possibility that under reporting of community-based melanoma cases in contrast to aggressive case-finding at LLNL could have contributed to the elevated rate found for LLNL. The investigators were at Stanford Univ. Medical School and the Northern California Cancer Center. They concluded that LLNL cases for the period 1974-1985 had thinner lesions than those in the community, thus allowing for some contribution from surveillance bias. However, the best estimate of the fraction of missed cases not recorded in the Tumor Registry was 12.7 percent, a fraction far too small to explain the observed three-fold elevation of the LLNL incidence rate.

Recent statistical analyses by Dr. Moore show that the LLNL incidence of invasive melanoma has declined gradually so that it is no longer significantly elevated above the rate in the surrounding counties. The rate for in situ cases continues to be elevated, but this might be expected in view of the dermatology clinic and frequent biopsy policy at LLNL.

Through the operation of the clinic Drs. Moore and Schneider have found what appear to be significant differences in clinical characteristics between invasive and in situ (non-invasive) cases (Lancet, 1990). The traditionally recognized constitutional factors for melanoma risk: hair and eye color, skin type, ability to tan vs burn in the sun, and various classifications of moles were all elevated in the invasive LLNL cases. However, none of the odds ratios for risk were elevated significantly in the in situ LLNL cases. This finding suggests a possible dichotomy in the population at risk for melanoma. the significance of which is at present unknown. On the basis of this work the data analysis in the Occupational Factors Study is presented both for the aggregated cases and 
controls and separately for invasive and in situ cases and their respective controls.

The LLNL Occupational Factors Study was a case-control study with one control matched to each case. All melanoma cases diagnosed among employees between January 1, 1969 (all cases prior to 1969 are deceased) and March 1, 1989 (the start of the study) were eligible for the study. The study included 69 cases who were alive and willing to participate. A "best-match" control (an LLNL employee without melanoma) was selected among those similar to each case according to five criteria: 1) sex, 2) age, 3) start date at LLNL, 4) years of education, 5) years of tenure at LLNL. Three methods were used to gather information from cases and controls. A questionnaire to assess constitutional factors, including many known risk factors for melanoma such as ethnicity, hair and eye color, reaction of skin to sunlight and episodes of sunburning, was administered by a nurse. Each case and each control received a thorough examination by a dermatologist who also counted all moles larger than $2 \mathrm{~mm}$ in diameter. Finally, an occupational interview focusing on exposures to the Austin and Reynolds factors was conducted by a former Hazards Control division leader with 40 years association with LLNL and LBL. Each interview was recorded by a certified shorthand reporter and the transcript of the interview was used by a panel of three experts in occupational exposures to assess exposures to suspected occupational agents.

\section{Results from Occupational Factors Study}

We found the usual associations between non-occupational factors and risk of melanoma. Cases were more likely to burn rather than tan, tended to have more moles than controls and were more likely to have had a mole removed or a previous nonmelanoma skin cancer. We found that tanning ability and the total number of moles larger than $2 \mathrm{~mm}$ in diameter, as assessed by the dermatologist, would correctly identify 49 cases in the 69 case-control pairs (71\% correct classification). If we restrict the classification to case-control pairs where the case had invasive melanoma, 33 of $39(85 \%)$ are correctly identified by these two factors. Only one invasive case is misclassified, since the remaining five case-control pairs are indistinguishable on these two factors. Results based on responses to the questionnaire are also good for classifying all case-control pairs based on four factors: tanning ability, amount of sunbathing between ages 15 
and 25 , sun avoidance during the ten years preceding diagnosis and a pastime of hiking. These responses correctly identify cases in 56 of 69 pairs (all types of melanoma). The same four factors with a measure of UV exposure based on residential history in place of sunbathing during ages $15-25$ correctly identify cases in 35 of 39 pairs ( 90 percent) with invasive melanoma. Four invasive cases are misclassified by these four factors. No occupational factors were significant for melanoma risk. Controls had greater exposure than cases to three of the Austin and Reynolds factors: ionizing radiation, Pacific Test Site and chemist duties; while cases had slightly greater exposure than controls for two factors: Site 300 and volatile photographic chemicals. None of these differences was statistically significant. Furthermore, a computerized review of the words used by cases and controls during the occupational interview failed to find significant differences in word frequencies for words associated with any of the Austin and Reynolds factors. A detailed analysis of job experience, both at LLNL and elsewhere, on potential for exposures to hazardous chemicals, ionizing radiation, or nonionizing radiation showed very close correspondence between cases and controls. These findings fail to support those reported by Austin and Reynolds. A possible explanation for the lack of corroboration must include the following: In our study controls were matched to cases for two important characteristics which were not used as matching criteria in the Austin and Reynolds study: years of education and start date of employment at LLNL. Austin and Reynolds reported, as have several other studies of melanoma, years of education as a significant risk factor for melanoma. The currently accepted hypothesis is that income increases with years of education and increased income leads to increased leisure activities in sunny areas. In fact, based on data from studies in Australia, it appears that exposure to intense UV during early teenage years is the most significant solar risk factor. It is believed that those with increased years of education likely come from families of higher socioeconomic status who could afford sunny vacations and college educations for their offspring. Thus, by failing to match for years of education, the Austin and Reynolds study confounded this risk factor with occupational factors. For example, chemist duties requires chemist education so that the risk factor chemist may be, at least in part, explained by educational background. Over the years the LLNL workplace has experienced increasing concern with exposure to both chemicals and ionizing radiation. This has resulted in a decrease in exposure over time so that employees with earlier start dates are likely to have 
higher exposures than those with later start dates. We can demonstrate that this is true in the 138 members of our case-control study. Thus, by failing to match for start date, Austin and Reynolds introduced a possible confounding of exposure factors. For example, if a case who started work at LLNL in the 1960 s is matched to a control who started in the 70 s, their exposures to chemicals and ionizing radiation would be expected to differ. We would be unable to determine whether a specific exposure or just generally higher exposure leads to increasing melanoma risk. On the other hand, when controls are matched to cases with respect to start date we can better determine whether specific exposures increase risk of melanoma. Overall, the recently completed LLNL study suggests that the occupational factors identified in the Austin-Reynolds case-control study may be the result of inadequate matching criteria that turn out to have been critical for the unique demography of the LLNL workforce. 


\section{Overall Conclusion on Increased Melanoma Incidence}

We agree with the findings that the rate of diagnosis of melanoma in LLNL employees was significantly elevated for a period of perhaps fifteen years; more recently the trend in incidence is decreasing so that the current rate for invasive melanoma is equal to the rate in the surrounding community. In situ cases remain elevated, presumably because of aggressive surveillance through continual education and the onsite clinic. Despite the assertion by Austin and Reynolds in 1984 that the elevated rate was caused by exposure to several occupational factors specific to employment at the Lab. , intensive investigation and review give no tangible support to this claim. We believe that the alleged factors arose from design flaws in case-control matching that were highly specific to our employee demographics.

The true cause of the elevated incidence of melanoma has not been determined. Some occupational exposures, especially those more prevalent in the early years of Laboratory operations and perhaps absent or under protective controls recently, cannot be ruled out. However, it seems unlikely that at this late date any further search for occupational factors would be rewarding. Unexplained clusters of cancer, including melanoma, have been recognized in many other populations; and this seems to be a possibility at LLNL. However, an hypothesis based upon some special epidemiological characteristics of melanoma is also appealing. Laboratory employees are generally well-educated, with many at the upper end of the range. They exhibit a propensity for intensive outdoor recreation, probably beginning in youth, but limited by work to sporadic rather than continuous solar exposure. And they live in the Bay Area, which has a higher incidence of melanoma than many other areas in the USA. From the 1950s through part of the 1980s outdoor recreation without much thought for protection from solar exposure became commonplace. If one couples the foregoing ideas with the concern by the Laboratory Health Services when a surprising number of cases were noted, and the aggressive case-finding that resulted thereafter, the LLNL experience might be considered as a very special artifact. One should remember that as many as one-third of the invasive cases correspond to the community background rate and need not exhibit major risk factors. 


\section{The SpotCheck Program}

In 1984, convinced of the validity of the increased incidence of melanoma at LLNL, the Task Group designed a plan of lab-wide communication and education and established a dermatology clinic within the Health Services Dept. that was staffed by a highly qualified dermatologist, Dr. Jeffrey Schneider, and accessed the dermatopathology services of Dr. Richard Sagebiel at UCSF. The importance of moles and the recognition of early melanoma was publicized in the news media. In addition a form was provided to all employees (and new employees on arrival) for self-counting moles over the entire body with reporting to the clinic. Employees considered to be at high risk on the basis of reported moles, or who considered themselves to be at risk for melanoma, were given clinic appointments for skin examination. Suspicious lesions were biopsied by a dermatologist. In the following ten years the program and clinic have resulted in the diagnosis of 62 new cases of melanoma and identification of 267 employees considered to be at elevated risk. Both groups are scheduled for periodic followup. The 267 at increased risk represent $3 \%$ of the current LLNL worker population. Whether this percentage is high or low compared to the surrounding community is at this time unknown. The onsite clinic is also providing valuable epidemiologic information on lesion thickness, an important prognostic criterion, on demographic and clinical data of invasive and in situ cases, on the life cycle of dysplastic moles, and on trends in melanoma mortality. Several reports based on this data have been assembled and published by Drs. Schneider and Moore (see References). The LLNL study population and SpotCheck program are considered to be one of the most important epidemiologic resources in the world by experts in the field. Current measures of control worldwide are focused on education, surveillance and early detection--areas in which LLNL has been one of the leaders.

\section{Recommendations for Future Management}

1. Continue the SpotCheck education and onsite clinic programs for the next four years in order that important data may be secured on mortality trend and precursor lesion life history. The most important objective is to obtain evidence 
whether an aggressive program of education and surveillance will decrease mortality from melanoma.

2. After this time an obligation still remains to the known cases and employees who are at high risk. The clinic could be continued at LLNL for a longer period. However, if this is not desired, we recommend that continued care of the cases and those at high risk can be transferred to health care providers, preferably by defining care standards within insurance contracts. For Kaiser Permanente, which has been LLNL's largest provider, this should be no problem because their Walnut Creek center has demonstrated excellent care and cooperation in the past.

3. The Occupational Factors Study (UCRL- ) was performed and analyzed by LLNL personnel. Its essentially negative findings should be publicly released to the participants, the LLNL workforce, and the public press. However, the study will require some form of expert validation. Two possible routes are suggested. The report might be submitted to Dr. John Lee of the University of Washington in Seattle or Dr. Tom Mack at USC, giving them the options of reassembling a Working Group as before or of conducting a mail review and returning the comments to LLNL. Alternatively, the University of California might be asked to invite non-UC experts in melanoma and occupational medicine in the West Coast area to review the report. There is also the possibility that DOE, either SAN or in Washington, may choose to intervene.

4. If no change occurs to disturb the current trend toward disappearance of excess melanoma incidence at LLNL, we see no further need for investigation of possible workplace factors. Continued gathering and publication of clinical and epidemiologic data should occur.

5. Unfortunately nearly all individuals with first-hand knowledge of the investigation of melanoma and the design of the SpotCheck program have now departed LLNL, except for one Hazards Control member of the Task Group (Jim Johnson). The residual melanoma program may not need much oversight beyond that provided by Dr. Schneider. However, an institution the size of LLNL, with many potentially hazardous materials and devices on site, should have a multidisciplinary organization like the Task Group to anticipate and cope 
effectively with future problems that might arise in the areas of employee health and safety. Ongoing activities of such a group would involve following and responding to health surveys by external entities oriented toward LLNL (two are currently being organized). Another function of a Task Group might be to design and to oversee a real-time, comprehensive health record database on employees which would be oriented toward early detection of clusters of illness based on work location or potential exposures. 